\title{
Combinatorial Biosynthesis: New Genetic Tools for Organic Synthesis of Polyketide Natural Products
}

\author{
Kenji Kinoshita \\ Department of Microbiology, School of Pharmaceutical Science, Toho University, \\ Received July 12, 2004
}

\begin{abstract}
During the past decade, modular multifunctional polyketide synthases (PKSs) have presented an exciting paradigm for the controlled genetic manipulation of complex natural product biosynthesis. These enzymes catalyze the biosynthesis of polyketide natural products by stepwise condensation and modification of metabolically derived building blocks such as malonyl-, methylmalonyl-, ethylmalonyl-, and methoxymalonyl-CoA. Many new tools for genetic manipulating and studying these multifunctional enzymes in vitro have been developed. The most significant recent advances in combinatorial biosynthesis, which is more rational and faster methods of engineering new compounds for the development of biological active agents from natural products, are reviewed.
\end{abstract}

\section{Introduction}

Polyketides are a large family of natural products mostly found as secondary metabolites in microbial fermentation, and they possess a wide variety of pharmacologically important activities. ${ }^{1}$ Recent literature on polyketide biosynthesis suggests that polyketide synthases (PKSs) have much greater diversity in both mechanism and structure, and is classified by the current Type I, II and III PKSs. The remainder of this review will be focused on the interplay between chemistry and biology to understand the Type I polyketide synthase structure and the mechanisms, and the application of this basic knowledge to solve the major problems associated with development of natural product drugs.

Modular Type I PKSs are large $\left(M_{\mathrm{r}} 100-1,000 \mathrm{kDa}\right)$ multifunctional enzyme systems that are responsible for the biosynthesis of structurally complex natural products from simple building blocks such as malonyl-, methylmalonyl-, ethylmalonyl-, and methoxymalonyl-CoA and are organized into coordinated groups of active sites, in which each module is responsible for one complete cycle of polyketide chain elongation and functional group modification. The polyketide chain-building step is a decarboxylative condensation reaction catalyzed by a $\beta$-ketoacyl-ACP synthase ( $\beta$ ketosynthase, KS) domain similar to that of the Claisen condensation reaction. ${ }^{2}$ Almost all PKS modules possess a core set of three domains: a $\beta$-ketosynthase (KS), an acyl transferase (AT), and an acyl carrier protein (ACP) domain. The degree of functional group modification taking place during each chain elongation cycle is determined by the specific combination of additional $\beta$-keto reductase (KR), dehydrase $(\mathrm{DH})$, and enoyl reductase (ER) domains found in each module. Modular Type I PKSs are responsible for the biosynthesis of the polyketide precursors of a wide variety of secondary metabolites including erythromy $\operatorname{cin}^{3}$, methymycin ${ }^{4}$, tylosin ${ }^{5}$, mycinamicin ${ }^{6}$, epothilone ${ }^{7}$, rapamycin ${ }^{8}$ and rifamycin ${ }^{9}$. The well-understood modular PKSs is 6-deoxyerythronolide B synthase (DEBS) from Saccharopolyspora erythraea, which catalyzes the formation of 6-deoxyerythronolide $\mathrm{B}(6-\mathrm{dEB}, 1)$, the parent 14 -membered macrolide aglycone of the broad-spectrum antibiotic erythromycin A (Figure 1). ${ }^{3,10}$ The formation of 6 -dEB (1) involves six condensation steps beginning with a propionyl-CoA starter unit and six methylmalonyl-CoA extender units. The six modules of DEBS are organized into three bimodular, homodimeric proteins, DEBS1, DEBS2, and DEBS3.$^{11}$ At the $N$-terminus of module 1 in DEBS1 is a specialized set of AT and ACP domains that together are responsible for loading of the propionyl-CoA primer unit. The $C$-terminus of DEBS3 carries a thioesterase (TE) domain that releases the heptaketide product from ACP6 by cyclization to the macrolactone 6-dEB (1).

\section{Combinatorial Biosynthesis Tools for Polyketide Natu- ral Products}

Although, homologous recombination is a particularly attractive strategy for manipulating extremely complex PKSs encoded by large gene clusters, the genetic engineering of PKSs in the chromosome of homologous organisms for microbial polyketides is often technically difficult and relatively slow because exogenous recombinant genes must be discriminated. Accordingly, an alternative strategy for combinatorial biosynthesis involves a heterologous cxprcssion system of selected PKS genes in suitable hosts. Additionally, the heterologous expression system offers the advantage of relatively easy access to PKS proteins through over expression similarly to those of wild strain. The production of the 6-dEB analog was made possible by a plasmid-based heterologous expression system developed for Streptomyces coelicolor, a model actinomycetes with a well-developed genetic tool. ${ }^{12}$ An improvement to this approach was achieved by concomitant expression of modular PKS genes from a multiple plasmid system developed for Streptomyces and was used to generate a library of 6-dEB analogs. ${ }^{13,14} \mathrm{Up}$ to the past few years, growth in the number of new PKS gene clusters has been rapidly witnessed and sequenced, and several manipulations of these have been adapted to heterologous expression systems, including the gene clusters encoding epothilone $e^{7}$, oleandomycin ${ }^{15}$, megalomicin ${ }^{16}$ and picromycin ${ }^{17}$. Increasingly, the activity in the field of combinato- 


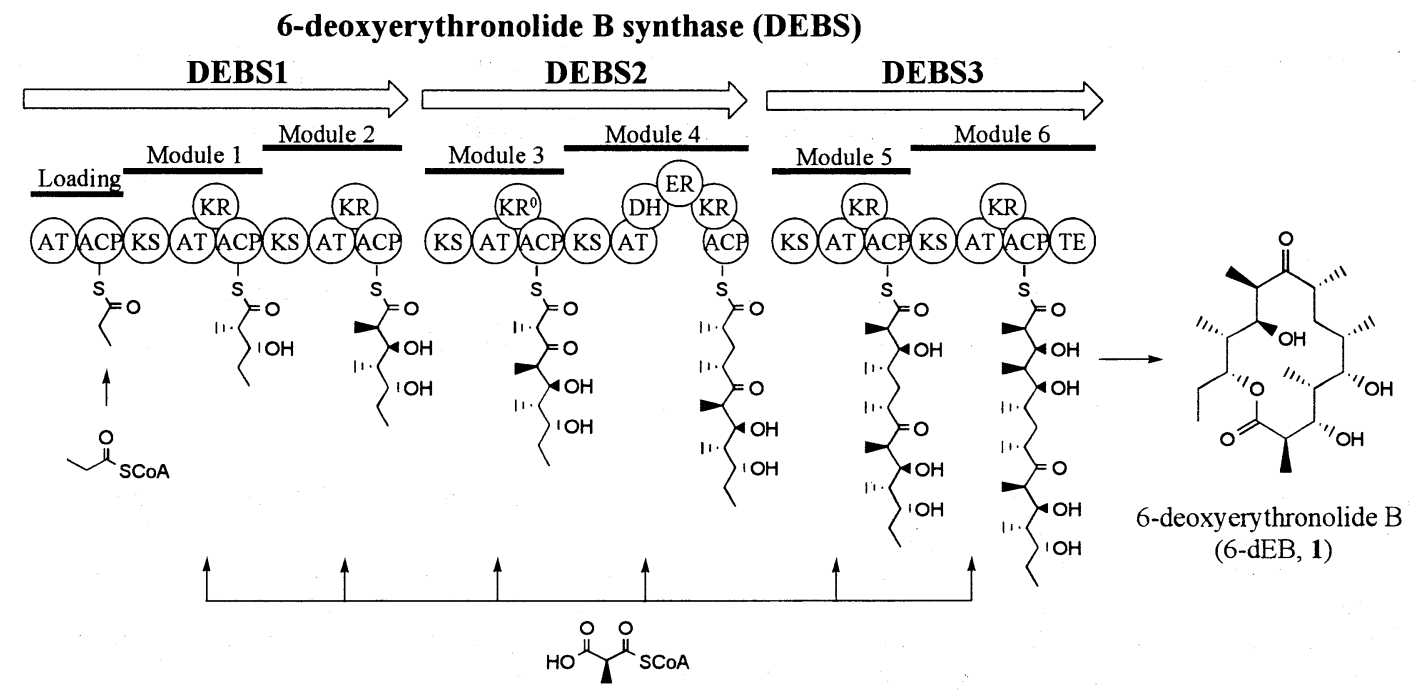

Figure 1. Polyketide Biosynthesis for Natural Products. Modular organization of DEBS. Each of the six modules contains a ketosynthase (KS), acyl transferase (AT), and acyl carrier protein (ACP), as well as variable numbers of supplementary domains, such as ketoreductase (KR), dehydrase (DH), and enoylreductase (ER). The loading didomain primes module 1 and a thioesterase (TE) domain cyclize the heptaketide and generate 6-dEB (1).

rial biosynthesis has also led to more widespread use of genetic tools in various actinomycetes hosts. Several new hosts for manipulation have subsequently been reported, including S. coelicolor, ${ }^{12}$ Streptomyces lividans ${ }^{18}$ and Streptomyces venezuelae ${ }^{19}$ that is the methymycin/picromycin producer. The most impressive advances in PKS expression host have occurred in gram-positive bacterial strain Escherichia coli, which has been used for the overexpression of PKS proteins in a number of in vitro studies. ${ }^{20}$ Moreover, the final 6-dEB (1) producing E. coli strain engineered by Pfeifer et $a l^{21}$ included the $s f p$ phosphopantetheinyl transferase gene $\mathrm{e}^{20}$ from an NRPS cluster in Bacillus subtilis, overexpression of the native prpE propionyl-CoA synthase, disruption of the propionyl-CoA catabolic pathway, and the $p c c$ propionylCoA carboxylase genes from $S$. coelicolor ${ }^{22}$ to convert propionyl-CoA to $(2 S)$-methylmalonyl-CoA. This significant technological achievement in E. coli promises to greatly accelerate the rate at which combinatorial biosynthesis tools can be developed. Indeed, when cultivated at high cell densities similar to that of Streptomyces, this derivative of $E$. coli BAP1 harboring DEBS1, 2 and 3 could accomplish titers of 6-dEB (1) up to $200 \mathrm{mg} / \mathrm{L} .{ }^{21}$ As those mentioned above, the use of biosynthetic genes from heterologous hosts suggests that other important polyketide precursors produced by fungus may also be expressed in E. coli.

\section{Biochemical Analysis of Modular Polyketide Synthases in vitro}

The situation of detailed mechanistic studies on microbial PKSs has changed dramatically over the past decade with several reports demonstrating enzymatic synthesis of polyketides using the PKS responsible for the polyketide component of the tetracenomycin synthase $e^{23}$, the truncated forms of modular PKSs in DEBS and the completed the DEBS assembly. $^{24,25}$ In the case of PKSs, some well-established questions about polyketide biosynthesis are as follows. How are their molecular recognition features cognizable among different subunits, domains, and modules? How tolerant is the interrelationship between the intrinsic substrate specificity of indi- vidual enzymes and the structures of their natural substrates? How strong is the discrimination of individual enzymes toward unnatural substrates?

The full DEBS proteins were achieved in a cell-free protein preparation from $S$. coelicolor $\mathrm{CH} 999 / \mathrm{pCK} 7 .{ }^{26}$ In the presence of propionyl-CoA, $(2 R S)$-methylmalonyl-CoA, and NADPH in a sodium phosphate buffer, the multifunctional enzyme assembly was found to catalyse the formation of 6-dEB (1) (Figure 2a). ${ }^{22}$ Analogous to the above studies with the complete DEBS system, more comprehensive investigations have also been carried out on the formation of the triketide lactone 2 by the truncated DEBS $1+$ TE protein preparation from $S$. coelicolor $\mathrm{CH} 999 / \mathrm{pCK} 9$. Substitution of acety1-CoA or butyry1-CoA for propiony1-CoA resulted in formation of the corresponding $\mathrm{C} 8$ - and $\mathrm{C} 10$-triketide lactone analogues 3 and $\mathbf{4}$ (Figure 2b). ${ }^{27,28}$ In competition experiments, DEBS $1+$ TE showed a 32 -fold preference in $k_{\text {cat }} / K_{\mathrm{m}}$ for propiony1-CoA over acety1-CoA. ${ }^{26,29}$ This kinetic preference, which must reflect individual rate constants up to and including the first irreversible enzymatic step, is most likely caused by the intrinsic rate of acylation of the active site serine of the AT loading domain. The use of full and partial modular polyketide synthases in specific reactions in combination with surrogate substrates has provided a powerful tool to produce novel analogues of medicinally important natural products by the engineered biosynthesis. Moreover, a major demand on the successful accomplishment of this promising tool is a better understanding of the structural and stereochemical features that influence the processing of substrate analogues by individual polyketide synthase modules. The individual DEBS modules 2, 3, 5, and 6 with the C-terminus of natural DEBS thioesterase (TE) domain have been expressed and purified from Escherichia coli. ${ }^{30}$ Incubation of (2S, 3R)-2-methyl-3-hydroxypentanoyl-SNAC diketide 5 with modules 2,5 , or $6+$ TE in the presence of methylmalonyl-CoA and NADPH resulted in formation of the triketide lactone 2 (Figure 2c), whereas incubation of 5 with module $3+$ TE gave rise to the 3-keto-triketide lactone for the inactivated KR3 (Figure 2d). The intrinsic substrate 
a)<smiles>CCNC(=O)CC</smiles>
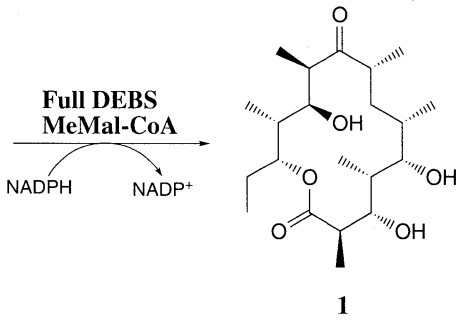

b)
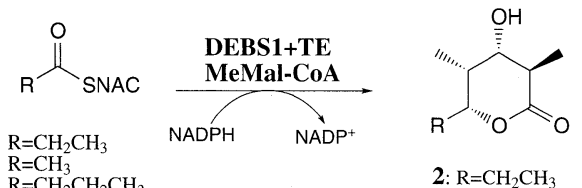

2: $\mathrm{R}=\mathrm{CH}_{2} \mathrm{CH}_{3}$

3: $\mathrm{R}=\mathrm{CH}_{3}$

4. $\mathrm{R}=\mathrm{CH}_{2} \mathrm{CH}_{2} \mathrm{CH}_{3}$

c)
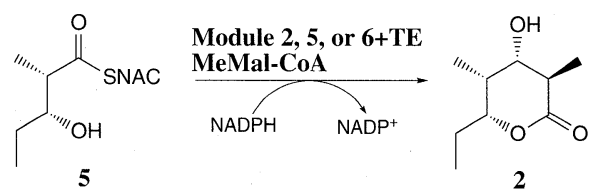

d)<smiles></smiles>
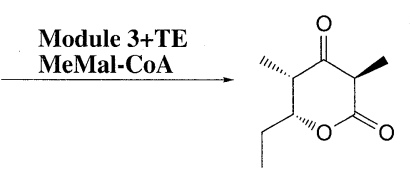

Figure 2. Tolerance specificity of the 6-deoxyerythronolide B synthase (DEBS) towards unnatural units. (a) 6-dEB catalyzed by Full DEBS. (b) triketide lactone 2, 3 and $\mathbf{4}$ by DEBS1 + TE. (c) triketide lactone 2 by individual module 2, 5, and 6. (d) 3-keto-triketide lactone by module 3 .

specificity on each of these recombinant modules has been also compared by the natural $(2 S, 3 R)$-2-methyl-3-hydroxypentanoyl-SNAC diketide $\mathbf{5}$ and its three diastereomers. ${ }^{29,31}$ In all cases, the syn-diketide $\mathbf{5}$ was the most preferred over its enantiomer as measured by the relative $k_{\text {cat }} / K_{\mathrm{m}}$ values. $^{31}$

Although the role of protein-protein communications is keenly appreciated in biological signal transduction processes such as the regulation of gene expression, it has been poorly explored in metabolic biochemistry, where individual enzymes are expected to have exquisite specificity for the products of the previous reaction. As the structural and mechanistic basis for these and other properties becomes clearer, this information will undeniably loop back to the design drawing-board through the emergence of a new generation of strategies for combinatorial biosynthesis. ${ }^{31}$

\section{Precursor-Directed Biosynthesis: Substrates Specificity and Tolerance}

Inactivation by site-directed mutagenesis of the KS1 domain that normally catalyzes the first condensation step of 6-dEB biosynthesis gives a mutant strain, DEBS $\left(\mathrm{KSl}^{\circ}\right)$, that no longer produces 6-dEB (1) (Figure 3a). Formation of 6-dEB (1) can be restored, however, when $(2 S, 3 R)$ 2-methyl-3-hydroxypentanoic acid-SNAC thioester (5), an $N$-acetylcysteamine thioester derivative of the natural diketide chain elongation intermediate, is administered to cultures of $\mathrm{S}$. coelicolor $\mathrm{CH} 999$ harboring plasmid $\mathrm{pJRJ} 2$ encoding DEBS $\left(\mathrm{KS}^{\circ}\right)^{32}$ Various diketide structural analogues modified in the starter unit $(6 \mathrm{a}-\mathrm{e})$ have also been used to prepare the corresponding 6 -dEB analogues $(7 \mathrm{a}-\mathrm{e})$ by using this DEBS $\left(\mathrm{KS}^{\circ}\right)$ mutant (Figure $\left.3 \mathrm{~b}\right) .^{32,33 \mathrm{~b}}$ The diastereomeric branched-chain diketide substrates 8 and 9 were also recognized by DEBS module $2 \mathrm{KS} 2$ and processed by the downstream modules to give the expected macrolactone products, the $(14 S)-13-s$-butyl $6-\mathrm{dEB}$ analogue 10 and the $(14 R)-$ 13-s-butyl 6-dEB analogue 11. Unexpectedly, feeding of the $3: 2(4 R: 4 S)$ mixture of 8 and $\mathbf{9}$ under the same conditions gave a single macrolide product, identical in all respects with the sample of $\mathbf{1 1}$ that had been obtained from the feeding of the $4 R$-branched-chain diketide thioester 9 alone (Figure 4). The biochemical basis for this stereochemical discrimination was established using purified DEBS module $2+$ TE to determine the steady-state kinetic parameters for $\mathbf{8}$ and $9 .^{33 \mathrm{~h}}$

a)

a) Streptomyces coelicolor CH999/pJRJ2 (DEBS KS10)

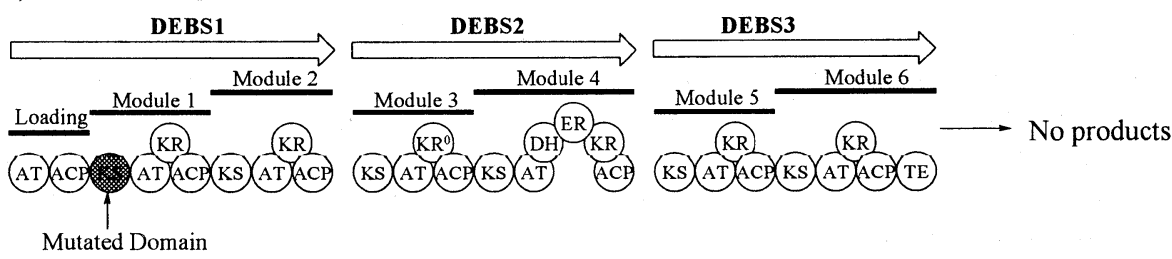

b)<smiles>[R2]C(O)[C@H]([R6])C(=O)NC(C)=O</smiles>

5,6

\section{S. coelicolor CH999/pJRJ2 \\ DEBS (KS1 ${ }^{0}$ )}

5, 1: $\mathrm{R}_{1}=\mathrm{CH}_{3}, \mathrm{R}_{2}=\mathrm{CH}_{2} \mathrm{CH}_{3}$ 6a, 7a: $\mathrm{R}_{1}=\mathrm{CH}_{3}, \mathrm{R}_{2}=\mathrm{CH}_{2} \mathrm{CH}_{2} \mathrm{CH}_{2} \mathrm{CH}_{3}$ 6b, 7b: $\mathrm{R}_{1}=\mathrm{CH}_{3}, \mathrm{R}_{2}=\mathrm{CH}_{2} \mathrm{C}_{6} \mathrm{H}_{5}$ 6c, 7c: $\mathrm{R}_{1}=\mathrm{CH}_{3}, \mathrm{R}_{2}=\mathrm{CH}=\mathrm{CH}_{2}$ 6d, 7d: $\mathrm{R}_{1}=\mathrm{CH}_{2} \mathrm{CH}_{3}, \mathrm{R}_{2}=\mathrm{CH}_{2} \mathrm{CH}_{3}$

6e, 7e: $\mathrm{R}_{1}=\mathrm{OCH}_{3}, \mathrm{R}_{2}=\mathrm{CH}_{2} \mathrm{CH}_{3}$

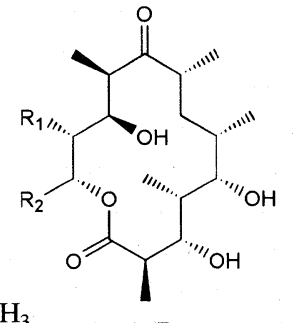

1,7

Figure 3. Precursor-directed biosynthesis by $S$. coelicolor $\mathrm{CH} 999$ harboring a plasmid encoding DEBS (KS1 $\left.{ }^{0}\right)$. a) Formation of 6-dEB (1) by DEBS $\left(\mathrm{KS}^{\circ}\right)$ is blocked but can be restored by addition of the diketide-SNAC 5. b) Conversion of various diketide structural analogs modified in the starter unit to the corresponding 6-dEB analogues. 


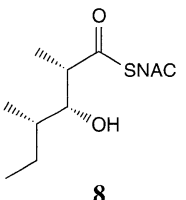

S. coelicolor

DEBS $\left(\mathrm{KS1}^{\mathbf{0}}\right)$<smiles>CC[C@H](C)[C@H](O)[C@@H](C)C(=O)[Mg]</smiles>

S. coelicolor DEBS $\left(\mathrm{KSI}^{\mathbf{0}}\right)$

9
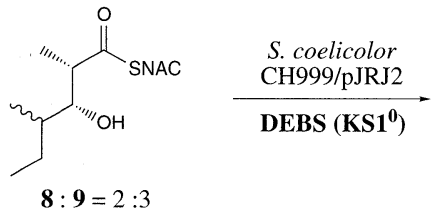
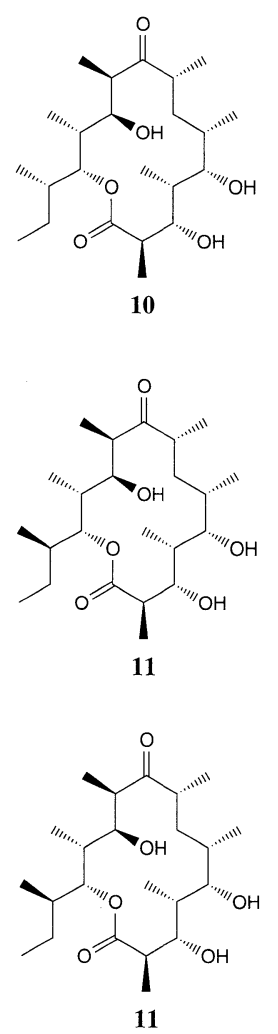

Figure 4. Conversion of branched chain diketide SNAC esters to 14-methyl-6-dEB analogues by $S$. coelicolor $\mathrm{CH} 999$ harboring DEBS $\left(\mathrm{KS}^{0}\right)$.

Furthermore, the anhydro-triketide thioester (12), an intermediate in the formation of tylactone, was also processed by the KS2 domain of DEBS $\left(\mathrm{KS}^{\circ}\right)$, resulting in the formation of the 16 -membered lactone (13), ${ }^{32}$ whereas the epimeric triketide thioester (14), with the natural erythromycin stereochemistry at $\mathrm{C}-4$, was apparently recognized as a triketide by KS3 and converted to the 10,11-anydro analogue of 6-dEB (15) (Figure 5). ${ }^{33 c}$

As mentioned above, the balance between intrinsic substrate specificity and tolerance for structural variations by individual KS domains holds the key to the exploitation of modular polyketide synthases for combinatorial biosynthesis. Such precursor-directed biosynthesis has provided a powerful tool to generate a wide range of novel polyketides by exploiting the apparently broad substrate specificity of DEBS KS2. Exogenous addition of diketide mimics to small-scale cultures of this null mutant resulted in highly selective multi-milligram production of unnatural polyketides. ${ }^{28}$ In particular, the unexpected incorporation of the presumed triketide intermediate as a diketide, and the cyclization of its fully elongated product into a 16-membered macrolactone, underscores the flexibility of the DEBS modules. The catalytic potential of this pathway could be harnessed by feeding to the organism a cell-permeable synthetic molecule that is suitably designed so as to be selectively loaded onto any desired PKS module at the corresponding KS domain. ${ }^{33 \mathrm{e}}$ Moreover, by utilizing surrogate diketide and triketide analogues, it is possible to exploit the apparently broad substrate specificity of the DEBS KS2 domain to generate a wide range of $6-\mathrm{dEB}$ derivatives. ${ }^{33}$ More recently, the versatility of this precursor-directed biosynthesis technology has been developed in the E. coli BAP1 harboring a DEBS mutant<smiles>CCC(C)[C@H](C)/C=C(\C)C(=O)OC</smiles>
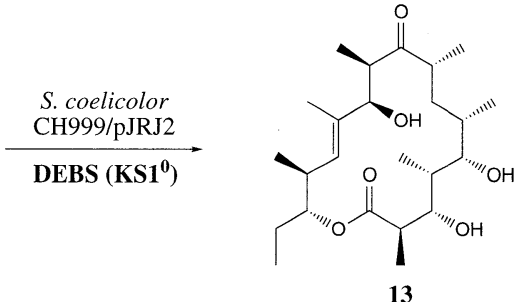

b)<smiles>CCC(C)[C@H](C)/C=C(\C)C(=O)OC</smiles>
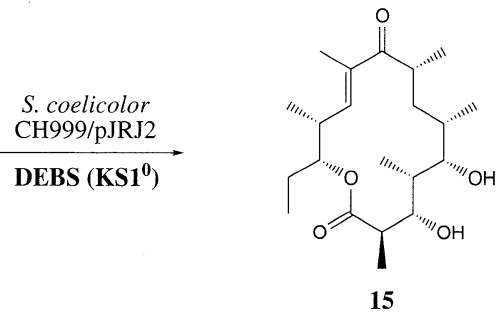

Figure 5. Precursor-directed biosynthesis of 14-membered and 16-membered macrolides. a) The administration of the diastereomeric the unsaturated triketide $\mathbf{1 2}$ is processed by DEBS module 2, resulting in the unexpected formation of 16-membered macrolactone 13. b) Feeding of the unsaturated triketide $\mathbf{1 4}$ results in formation of the corresponding 10,11-anydro analog of 6-dEB (15). Triketide 14 is recognized and processed as a triketide by DEBS module 3 .

from which the entire loading didomain and DEBS module 1 have been deleted. The unsaturated triketide thioesters $\mathbf{1 2}$ and 14, however, were not incorporated and converted to the expected macrolactone products 13 and 15 by the engineered $E$. coli strain, possibly reflecting uptake and /or metabolic differences between $E$. coli and S. coelicolor. ${ }^{33 \mathrm{~g}}$

\section{Genetic Manipulation of Modular Polyketide Biosyn- thesis}

The availability of the cloned DEBS structural genes opened the door to genetic modification of the DEBS proteins themselves. As shown in Figure 6, the pioneering demonstration by Katz et al. that KS domains might be able to accept modifications in their natural substrates evolved from the finding that mutagenic inactivation of the KR5 or ER4 domains of DEBS led to the formation of the corresponding 5-keto-6dEB $(\mathbf{1 6})^{34}$ and 6,7-anhydro-6dEB $(\mathbf{1 7})^{35}$ derivatives, respectively. These results indicated that the KS6 domain and all other domains in module 6 as well as the TE could accept the unreduced $\beta$-ketoacyl precursor of its natural $\beta$-hydroxyacyl hexaketide substrate, whereas the KS5 and KS6 domains would tolerate the corresponding enoylacy 1ACP analogue of the natural reduced pentaketide intermediate. A further impressive demonstration by KOSAN's researchers is that a combinatorial library of 6-dEB analogs was produced by engineering simultaneous changes in multiple modules of DEBS. ${ }^{36}$ The corresponding novel macrolides were produced with up to three modifications at one or more carbon centers, demonstrating that multiple changes in a polyketide pathway could be carried out. The 6-deoxyerythronolide B synthase (DEBS) from Saccharopolyspora erythrae $^{37,38}$ and the heterologous host of $S$. lividans ${ }^{36}$ have been the most widely used system to create enzymes that synthesize new molecules and have served as a model system for discovering ways to engineer PKSs and exploring the structural plasticity and substrate tolerance of modular PKSs. 
a) Saccharopolyspora erythraea DEBS (KR50)

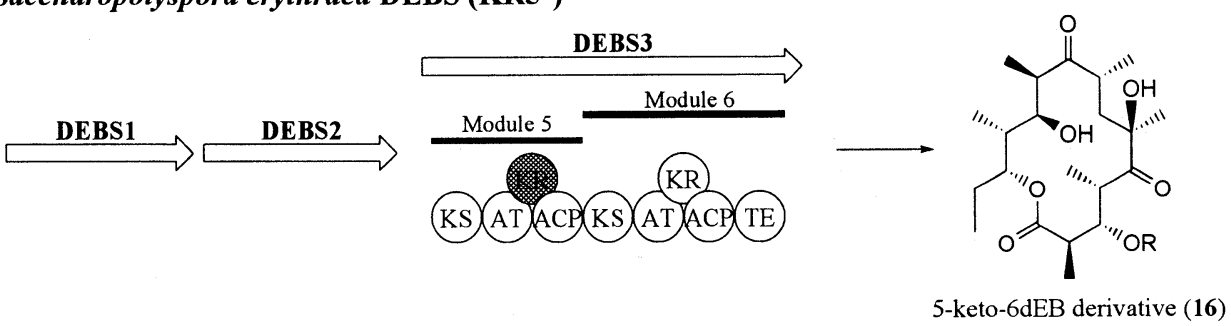

b) Saccharopolyspora erythraea DEBS (ER4 ${ }^{0}$ )

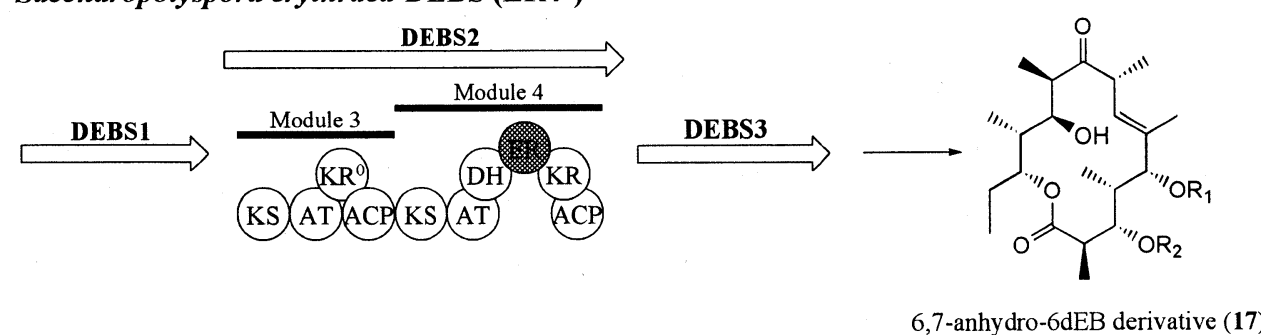

Figure 6. Tolerance of downstream modules in DEBS towards polyketide chains with altered degrees of reduction.

These experiments with DEBS generally involved the deletion, substitution or addition of individual domains or modules, or the creation of hybrid modules from different PKSs. ${ }^{10,39}$

Progress of combinatorial biosynthesis has been made in the understanding of how protein-protein interactions direct polyketide chain transfer between modules, an important consideration for the design and construction of hybrid PKSs. Expression of DEBS modules in E. coli and in vitro characterization led to the elucidation of short intermodular and interpolypeptide linkers as crucial components of PKS assembly. ${ }^{40}$ Thus, it was found that interpolypeptide linkers at the amino and carboxy termini of successive noncovalent neighboring modules are distinct from the intermodular linkers between modules in a single polypeptide and that the utility of an appropriate linker can allow heterologous modules to be functionally connected. ${ }^{31,40}$ Whole PKSs subunits encoding multiple modules from the DEBS, picromycin and oleandomycin were eventually shown to complement each other, indicating good communication between noncovalent heterologous modules in structurally related PKSs could exist. ${ }^{41}$ As shown in Figure 7, both genetically mutated strains could produce the same 14-membered macrolactone, $3,3^{\prime}$-dihydro-narbonolide. This approach was combined with the previous approach of individual domain manipulation to generate several new macrolide aglycone structures. ${ }^{41}$

\section{Conclusion and Perspectives}

The past decade has witnessed an exponential growth rate of genetic information on polyketide biosynthesis in general. The PKSs paradigms have been and will continue to be the cornerstones for polyketide biosynthesis. At present, the engineering of PKS to produce new compounds might be almost a routine task for the experienced chemist and microbiologist. The fact that new polyketides numbering more than two hundred compounds have been reported indicates that combinatorial biosynthesis is a relatively robust technology. Most of these compounds would have been difficult or impossible to construct through chemical synthesis. The combinatorial biosynthesis toolbox is becoming increasingly large

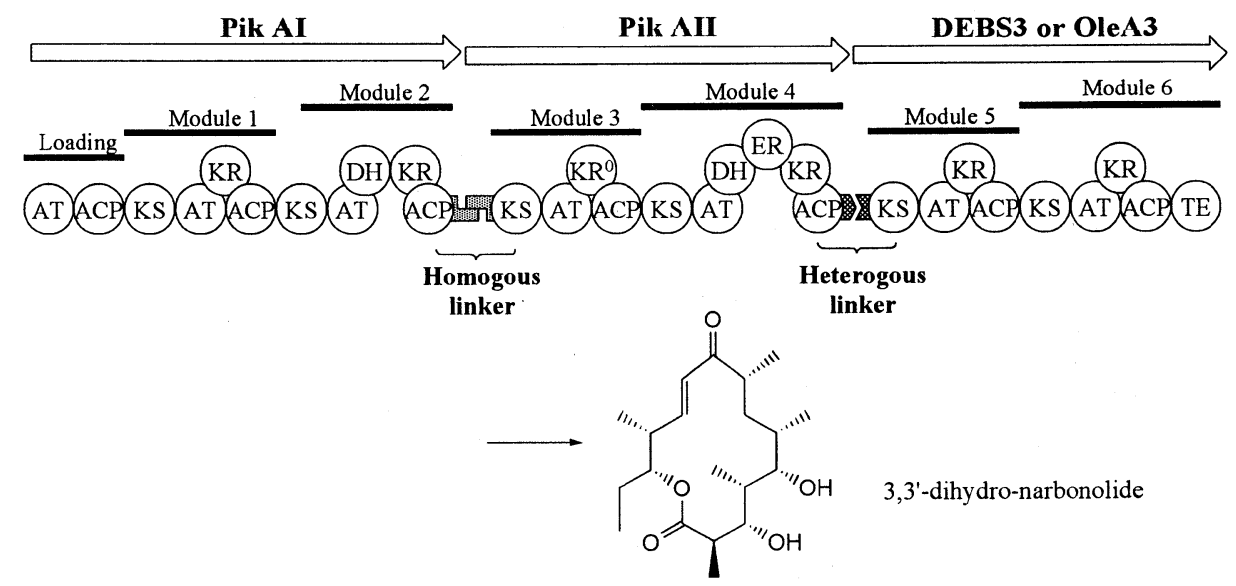

Figure 7. Directed chain transfer between modules and subunits in modular PKSs. Complete subunits encoding multiple modules from heterologous sources can be used in functional complementation. PKS subunits from the picromycin PKS (PikAI and PikAII) were combined with DEBS (DEBS3) or oleandomycin PKS (OleA3) subunits. It is interesting that, in these cases, non-cognate interpolypeptide linkers remained compatible. 
and additional ways to engineer novel polyketide natural products continue to be developed. Thus, the novel biosynthetic study field that may be on the horizon is the union of combinatorial chemistry and combinatorial biosynthesis. Finally, the utility of this genetical technology to create useful pharmaceuticals or lead compounds should be welldemonstrated in the next few years. Also, my personal dream over the past two decades to produce "unnatural" natural products for mycinamicin will comes true. ${ }^{6}$

Acknowledgments I am grateful to Professor David E. Cane at Brown University for helpful discussion and critical comments on this manuscript, and to his co-workers and collaborators who have made dreams into reality through their diligence and dedication. I would also like to thank Professors Fumio Kato and Yujiro Anzai at Toho University for providing the opportunity to meet the challenge of combinatorial biosynthesis of mycinamicin.

\section{References}

1) (a) O'Hagan, D. O. The Polyketide Metabolites; Ellis Norwood; New York, 1991. (b) Cane, D. E. Polyketide and Nonribosomal Polypeptide Synthesis. Chem. Rev. 1997, 97(7).

2) (a) Cane, D. E.; Liang, T.-C.; Taylor, P. B.; Chang, C.; Yang, C.-C. J. Am. Chem. Soc. 1986, 108, 4957. (b) Weissman, K. J.; Timoney, M.; Bycroft, M.; Grice, P.; Hanefeld, U.; Staunton, J.; Leadlay, P. F. Biochemistry. 1997, 36, 13849.

3) (a) Donadio, S.; Staver, M. J.; Mcalpine, J. B.; Swanson, S. J.; Katz, L. Science 1991, 252, 675. (b) Cortes, J.; Haydock, S. F.; Roberts, G. A.; Bevitt, D. J.; Leadlay, P. F. Nature 1990, 348, 176.

4) (a) Xue, Y.; Zhao, L.; Liu, H.-W.; H.Sherman, D. Proc. Natl. Acad. Sci. USA 1998, 95, 12111. (b) Tang, L.; Fu, H.; Betlach, M. C.; McDaniel, R. Chem. Biol. 1999, 6, 553.

5) (a) Baltz, R. H.; Seno, E. T.; Annu. Rev. Microbiol. 1988, 42 , 547. (b) DeHoff, B. S.; Sutton, K. L.; Rosteck, P. R., Jr. Genbank, Accession Number U78289 1997.

6) (a) Kinoshita, K.; Takenaka, S. Hayashi, M. J. Chem. Soc. Chem. Commun. 1988, 1988, 943. (b) Anzai, Y.; Saito, N.; Tanaka, M.; Kinoshita, K.; Koyama Y.; Kato F. FEMS Microbiol. Lett. 2003, 218, 135.

7) (a) Julien, B.; Shah, S.; Ziermann, R.; Goldman, R.; Katz, L.; Khosla, C. Gene 2000, 249, 153. (b) Tang, L.; Shah, S.; Chung, L.; Carney, J.; Katz, L.; Khosla, C.; Julien, B. Science 2000, 287, 640.(c) Molnár, I.; Schupp, T.; Ono, M.; Zirkle, R. E.: Milnamow, M.; Nowak-Thompson, B.; Engel, N.; Toupet, C.; Strattmann, A.; Cyr, D. D.; Gorlach, J.; Mayo, J. M.; Hu, A.; Goff, S.; Schmid, J.; Ligon, J. M. Chem. Biol. 2000, 7, 97.

8) Schwecke, T.; Aparicio, J. F.; Molnár, I.; König, A.; Khaw, L. E.; Haycock, S. F.; Oliynyk, M.; Caffrey, P.; Cortes, J.; Lester, J. B.; Böhm, G. A.; Staunton, J.; Leadlay, P. F. Proc. Natl. Acad. Sci. U. S. A. 1995, 92, 7839.

9) (a) August, P. R.; Tang, L.; Yoon, Y. J.; Ning, S.; Muller, R.; Yu, T. W.; Taylor, M.; Hoffmann, D.; Kim, C. G.; Zhang, X.; Hutchinson, C. R.; Floss, H. G. Chem. Biol. 1998, 5, 69. (b) Schupp, T.; Toupet, C.; Engel, N.; Goff, S. FEMS Microbiol. Lett. 1998, 159, 201.

10) (a) Katz, L. Chem. Rev. 1997, 97(7), 2557. (b) Khosla, C.; Gokhale, R. S.; Jacobsen, J. R.; Cane, D. E. Ann. Rev. Biochem. 1999, 68, 219. (c) Staunton, J.; Wilkinson, B. in Comprehensive Natural Products Chemistry, Polyketides and Other Secondary Metabolites Including Fatty Acids and Their Derivatives; Ed. Sankawa, U. Vol. Ed. Barton, D.; Nakanishi, K.; Meth-Cohn, O. Elsevier, Oxford, 1999, Vol. 1, pp. 495-532. (d) Staunton, J.; Weissman, K.J. Nat. Prod. Rep. 2001, 18, 380.

11) Caffrey, P.; Bevitt, D. J.; Staunton, J.; Leadlay, P. F. FEBS Lett. 1992, 304, 225

12) McDaniel, R.; Ebert-Khosla, S.; Hopwood, D.; Khosla, C. Sci- ence 1993, 262, 1546.

13) Xue, Q.; Ashley, G.; Hutchinson, C. R.; Santi, D. V. Proc Natl Acad Sci USA 1999, 96, 11740.

14) Ziermann R.; Betlach, M. J Ind Microbiol. Biotechnol. 2000, 24, 46.

15) Shah, S.; Xue, Q.; Tang, L.; Carney, J. R.; Betlach, M.; McDaniel, R. J Antibiot 2000, 53, 502.

16) Volchegursky, Y.; Hu, Z.; Katz, L.; McDaniel, R. Mol Microbiol. 2000, 37, 752 .

17) Tang, L.; Fu, H.; Betlach, M. C.; McDaniel, R. Chem. Biol. $1999,6,553$

18) Ziermann, R.; Betlach, M. C. Biotechniques 1999, 26, 106.

19) (a) Xue, Y.; Zhao, L.; Liu, H. W.; Sherman, D. H. Proc Natl Acad Sci USA 1998, 95, 12111. (b) Zhao, L.; Sherman, D. H.; Liu, H. W. J. Am. Chem. Soc. 1998, 120, 10256. (c) Zhao, L.; Ahlert, J.; Xue, Y.; Thorson, J. S.; Sherman, D. H.; Liu, H. W. J. Am. Chem. Soc. 1999, 121, 9881. (d) Chen, S.; Xue, Y.; Sherman, D. H.; Reynolds, K. A. Chem. Bio. 2000, 7, 907.

20) Kealey, J. T.; Liu, L.; Santi, D. V.; Betlach, M. C.; Barr, P. J. Proc. Natl. Acad. Sci. USA 1998, 95, 505.

21) Pfeifer, B. A.; Admiraal, S. J.; Gramajo, H.; Cane, D. E.; Khosla, C. Science 2001, 291, 1790.

22) Rodríguez, E.; Gramajo, H. Microbiol. 1999, 145, 3109

23) Shen, B.; Hutchinson, C. R. Science, 1993, 262, 1535.

24) Pieper, R.; Luo, G.; Cane, D. E.; Khosla, C. Nature 1995, 378, 263.

25) Wiesmann, K. E. H.; Cortes, J.; Brown, M. J. B.; Cutter, A. L.; Staunton, J.; Leadlay, P. F. Chem. Biol. 1995, 2, 583.

26) Pieper, R.; Ebert-Khosla, S.; Cane, D. E.; Khosla, C. Biochemistry 1996, 35, 2054

27) Wiesmann, K. E. H.; Cortes, J.; Brown, M. J. B.; Cutter, A. L.; Staunton, J.; Leadlay, P. F.; Chem. Biol. 1995, 2, 583.

28) Pieper, R.; Luo, G.; Cane, D. E.; Khosla, C. J. Am. Chem. Soc. 1995, 117, 11373.

29) Chuck, J.; McPherson, M.; Huang, H.; Jacobsen, J. R.; Khosla, C.; Cane, D. E. Chem. Biol. 1997, 4, 757.

30) Gokhale, R. S.; Tsuji, S. Y.; Cane, D. E.; Khosla, C. Science 1999, 284,482 .

31) (a) Wu, N.; Kudo, F.; Cane, D. E.; Khosla, C. J. Am. Chem. Soc. 2000, 122, 4847. (b) Wu, N.; Tsuji, S. Y.; Cane, D. E.; Khosla, C. J. Am. Chem. Soc. 2001, 123, 6465. (c) Tsuji, S. Y.; Cane, D. E.; Khosla, C. Biochemistry 2001, 40, 2326. (d) Wu, N.; Cane, D. E.; Khosla, C. Biochemistry 2002, 41, 5056.

32) Jacobsen, J. R.; Hutchinson, C. R.; Cane, D. E.; Khosla, C. Science 1997, 277, 367.

33) (a) Jacobsen, J. R.; Keatinge-Clay, A.; Cane, D. E.; Khosla, C. Bioorg. Med. Chem. Lett. 1998, 6, 1171. (b) Jacobsen J. R.; Cane D. E.; Khosla, C. J. Am. Chem. Soc. 1998, 120, 9096. (c) Hunziker, D.; Wu, N.; Kinoshita, K.; Cane, D. E.; Khosla, C. Tetrahedrun Lett. 1999, 40, 635. (d) Kinushita, K.; Williard, P. G.; Khosla, C.; Cane, D. E. J. Am. Chem. Soc. 2001, 123, 2495. (e) Cane, D. E.; Kudo, F.; Kinoshita, K.; Khosla, C. Chem. Biol. 2002, 9, 131. (f) Kinoshita, K.; Pfeifer, B. A.; Khosla, C.; Cane, D. E. Bioorg, Med. Chem. Lett. 2003, 13, 3701. (g) Kinoshita, K.; Khosla, C.; Cane, D. E. Helvetica Chimca Acta 2003, 86, 3889.

34) Donadio, S.; Staver, M. J.; McAlpine, J. B.; Swanson, S. J.; Katz L. Science 1991, 252, 675.

35) Donadio, S.; McAlpine, J. B.; Sheldon, P. J.; Jackson, M.; Katz L. Proc. Nail. Acad. Sci. USA 1993, 90, 7119.

36) McDaniel, R.; Thamchaipenet, A.; Gustafsson, C.; Fu, H.; Betlach, M.; Ashley, G. Proc. Natl. Acad. Sci. USA 1999, 96, 1846.

37) Donadio, S.; Staver, M. J.; McAlpine, J. B.; Swanson S. J.; Katz, L. Science 1991, 252, 675.

38) Cortés, J.; Haydock, S. F.; Roberts, G. A.; Bevitt, D. J.; Leadlay, P. F. Nature 1990, 348, 176.

39) Katz, L.; McDaniel, R. Med. Res. Rev. 1999, 19, 543.

40) Gokhale, R. S.; Tsuji, S. Y.; Cane, D. E.; Khosla, C. Science 1999, 284, 482

41) Tang, L.; Fu H.; McDaniel, R. Chem. Biol. 2000, 7, 77. 
Kenji Kinoshita (born 1953) is currently a Managing Director in DNA Chip Research Inc., a Visiting Associate Professor of Medical Research Institute at Tokyo Medical and Dental University and Visiting Researcher of the School of Pharmaceutical Science at Toho University. After receiving his M.Sc. in 1980 from Kwansei Gakuin University, he joined Toyo Jozo Co., which was a brewery and pharmaceutical company. He received his Ph.D. in 1990 from Kwansei Gakuin University. He carried out polyketide biosynthetic research as an Assistant Professor of Chemistry with Professor David E. Cane at Brown University from 1997 to 2002. His current research interests are in the areas of the combinatorial biosynthesis of natural products. 